\title{
Intraoral Approach for Reduction and Fixation of Infraorbital Rim Fracture
}

Original Article

\author{
Abdullah Atef Hammuda
}

Oral and Maxillofacial Surgery Department,Faculty of Dentistry, Minia University

\begin{abstract}
Various surgical approaches are widely used for exposure, evaluation, and treatment of orbital trauma and pathology in the orbital floor and infraorbital rim. They were conveniently categorized as either transcutaneous or transconjunctival. The purpose of this research is to assess the intraoral approach for reduction and fixation of the infraorbital rim in zygomatic fractures. Patients and methods: 11 patients were subjected to reduction and fixation of infraorbital rim fracture through intraoral vestibular approach. Time of surgery, Post-operative edema and infraorbital nerve sensory function were evaluated. All patients were followed up for six months.

Results: all cases included in the current study did not show any lower eyelid complications . postoperative numbness and edema were reported and subcided completely .

Conclusion: Intraoral unilateral vestibular approach allows access to the zygomatico-maxillary buttress and infraorbital rim with only 1 incision, optimizes surgical time, decreases complication rates, and avoids periorbital scarring in the patient.
\end{abstract}

Key Words: Infraorbital rim, maxillofacial trauma, orbital fracture, zygomaticomaxillary complex

Received: 22 November 2018, Accepted: 25 December 2018

Corresponding Author: Abdullah Atef Hammuda, Lecturer of oral and maxillofacial surgery, Minia University, Egypt, E-mail: dr.abdullahatef@gmail.com

ISSN: 2090-097X, October 2018, Vol. 9, No. 4

\section{INTRODUCTION:}

The zygoma is presented with four projections creating a quadrangular shape. It articulates with four bones: the frontal, temporal, maxilla, and sphenoid when the zygoma is separated from its four articulations it is called a zygomatic complex fracture. Zygomatic arch may be fractured independently or as part of a zygomatic complex fracture. It connects the temporal process of the zygoma and the zygomatic process of the temporal bone. The infraorbital nerve (IO) passes through the orbital floor and exits at the infraorbital foramen. It provides sensation to the anterior cheek, lateral nose, upper lip, and maxillary anterior teeth. Muscles of facial expression originating from the zygoma include the zygomaticus major and labii superioris. They are innervated by cranial nerve VII. ${ }^{[1]}$

Zygomatic complex fracture is one of the most common facial trauma that has been described in the literature. However, there are several controversies regarding the best technique and treatment of these fractures, such as closed versus open reduction; the surgical approach; sequence of reduction and fixation in open techniques, complications, and morbidity rates of each technique. ${ }^{[2]}$

The intraoral approach for surgical treatment of zygomatic arch fractures was first described by Keen in 1909 , using the maxillary vestibular technique. Later, other studies showed different variations of this technique to reach the zygomatic arch and buttress. However, in most cases, infraorbital margin fixation was still performed through the subtarsal, subciliary, or transconjunctival approach.

Surgical approaches were conveniently categorized as either transcutaneous or transconjunctival. When performing surgery in this area, cosmetic concern is the major factor. ${ }^{[3,4]}$

It was stated that the transconjunctival approach allows adequate exposure of the orbital floor, has a low complication rate, and results in better cosmetic results as the incision is hidden within the inferior fornix of the eyelid. ${ }^{[5]}$

The advantages of the transconjunctival approach for orbital access include minimal scarring and a lower risk of eyelid displacement compared with other approaches. For that reason it was recommended that transconjunctival approach for orbital exposure is a safe and reliable method ${ }^{[6]}$

In most of cases of zygomaticomaxillary buccal vestibular incision is the first incision as it allows for exposure of the zygomaticomaxillary buttress, which is also the most commonly affected buttress of the zygomatic complex fracture. Other studies showed different variations of this technique to reach the body of zygomatic and zygomatic arch. However, it was claimed that intraoral approach lack for the accessibility to reach the infraorbital $\operatorname{rim} .^{[7,8]}$ 
If a modification or further dissection allow for exposure, reduction and fixation of the infraorbital rim it will be a god idea. The purpose of this article was to evaluate the intraoral approach for reduction and fixation of the infraorbital rim in zygomatic fractures.

\section{PATIENTS AND METHODS:}

In the present study prospective non controlled clinical study on eleven patients were selected from the department of oral and maxillofacial surgery, faculty of dentistry, Minia university and Dar Al-Shifa hospital of Ministry of Health, Egypt. All patients were suffering from unilateral zygomaticomaxillary complex fracture.

Inclusion criteria included were patients above the age of 15 years unilateral Zygomaticomaxillary complex fracture with infraorbital rim involvement. Exclusion criteria were orbital fractures where additional procedure required for reconstruction of orbital floor and presence of severe facial lacerations or orbital injuries

All cases were evaluated according to the advanced trauma life-support protocol. The examination was conducted with a cervical spine precaution protocol with evaluation of the cranial nerves, eyes, ears, and scalp. The face is then inspected and palpated for asymmetry caused by displaced fragments of the facial skeleton and for areas of oedema, ecchymosis, and lacerations. Oral examination was also conducted for ecchymosis or laceration at the zygomaticomaxillary interface. Dental occlusion and the integrity of the palate was evaluated. All cases were subjected to preoperative computed tomography (C.T.) scanning with $3 \mathrm{D}$ reconstruction.

Under general anesthesia intraoral approach to access the infraorbital rim was performed through a vestibular incision extending from the midline to the first molar, the mucoperiostium flap was elevated exposing anterior wall of maxilla, lateral wall of the nose and the piriform aperture to obtain greater motion of the flap. A special attention for the infraorbital nerve preservation as the infraorbital nerve epineurium was dissected to facilitate retraction as well as to tunnelize the infraorbital rim approach above the infraorbital foramen. A part of masseter and zygomaticus major muscles were detached from the inferior border of the zygoma and the zygomatic process of the maxilla exposing the zygomatic body and a part of zygomatic arch. After such exposure it is possible to access the infraorbital rim, to check the reduction in this region. Miniplate was preadapted over the skin then final adaptation was done on the infraorbital rim. Drilling and screw fixation was then performed. (Figure 1)

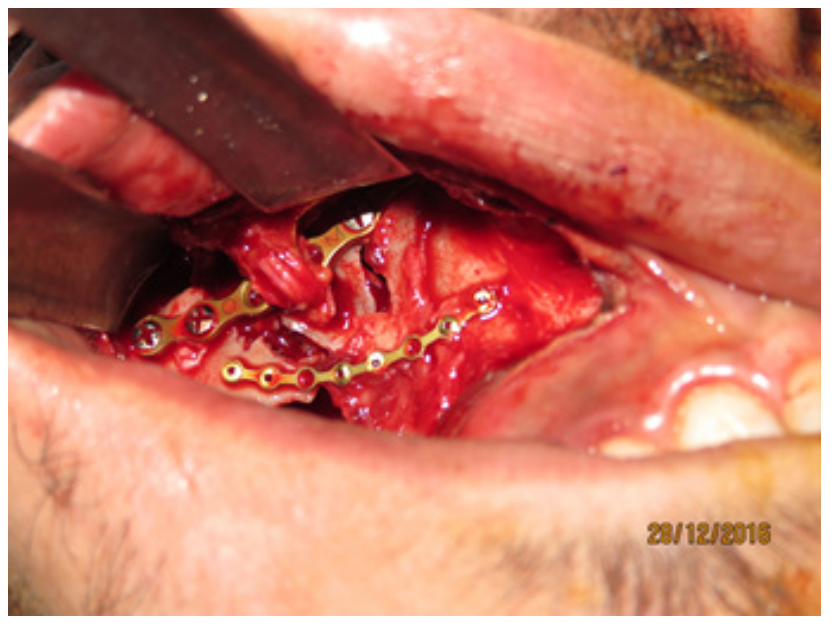

Fig.1: Image showing intraoral approach of fracture line with reduction and fixation of infraorbital rim

The time of surgery was measured since vestibular incision till complete exposure of infraorbital rim using stopwatch.

Post-operative edema was determined by the distance in millimetre between following landmarks; tragus point to angle of the mouth, Lateral canthus tragus line and tragus to pogonion line. ${ }^{[9]}$ The sum of three lines was considered the edema measure. It was recorded preoperatively, 1 day, 1 week, 1 month, 3 months and 6 months postoperatively.

difference between preoperative and postoperative summation of a 3 line measurements using 4 fixed points on surgical side of the face and finding the average. The fixed points used were $\mathrm{A}$; the most posterior point on the tragus, $\mathrm{B}$; lateral canthus of the eye, $\mathrm{C}$; the most lateral point on the angle of the mouth, and $\mathrm{D}$; most inferior point on the angle of the mandible. The 3 lines were $A B, A C$ and $\mathrm{BD}$.

Infraorbital nerve sensory functions were evaluated by two points discrimination test and numbness test. Two points discrimination was examined with 2 points sharp pointed calliper, the tests consists of alternating series with either ascending or descending increments with a successively longer or shorter pin distance in the device, during which the patient reported on a presence of sensation of two separate points of stimulation in comparison with contralateral side. A test series was terminated after a response reversal, i.e. when a particular type of response (positive/negative) on successive increments. ${ }^{[10]}$ Nerve numbness was assessed by subjective method by asking the patient about if there was a numbness in lower palpebral, nasal skin and upper lip. 
All patients were subjected to a follow up for six months.

Recorded data were analysed using the statistical package for social sciences, version 20.0 (SPSS Inc., Chicago, Illinois, USA).

\section{RESULTS:}

Selected patients were within age range 19 - 49 (average $29 \pm 10$ ). Traffic accident was the main reason of trauma in nine cases $81.8 \%$, assult in one case $9.09 \%$ and falling in one case $9.09 \%$. Cases presented with Zygomaticomaxillary complex fracture associated with other fractures have been shown in (Table 1)

Table1: Demography of patient age, Associated factured bone and Etiology of fracture

\begin{tabular}{cclc}
\hline Cases & Age & \multicolumn{1}{c}{ Associated factured bone } & $\begin{array}{c}\text { Etiology of } \\
\text { fracture }\end{array}$ \\
\hline 1 & 18 & Zmc + nasal bone & RTA \\
2 & 22 & Zmc + mandible & RTA \\
3 & 23 & Zmc + zygomatic arch & RTA \\
4 & 30 & Zmc + anterior wall of maxillary sinus & RTA \\
5 & 32 & Zmc + anterior wall of maxillary sinus & ASSULT \\
6 & 24 & Zmc + anterior wall of maxillary sinus & RTA \\
7 & 45 & Zmc + maxillary dentoalveolar bone & RTA \\
8 & 49 & Zmc + zygomatic arch & FALL \\
9 & 19 & Zmc + anterior wall of maxillary sinus & RTA \\
10 & 27 & Zmc + zygomatic arch & RTA \\
11 & 30 & Zmc + anterior wall of maxillary sinus & RTA \\
\hline
\end{tabular}

In general vision, pupil size, reactivity and eyeball movements were examined preoperatively and after recovery with no change or affection reported. No eyelid complications were reported. In addition, postoperative CT scans were performed for evaluation of reduction and surgery. (Figure 2)

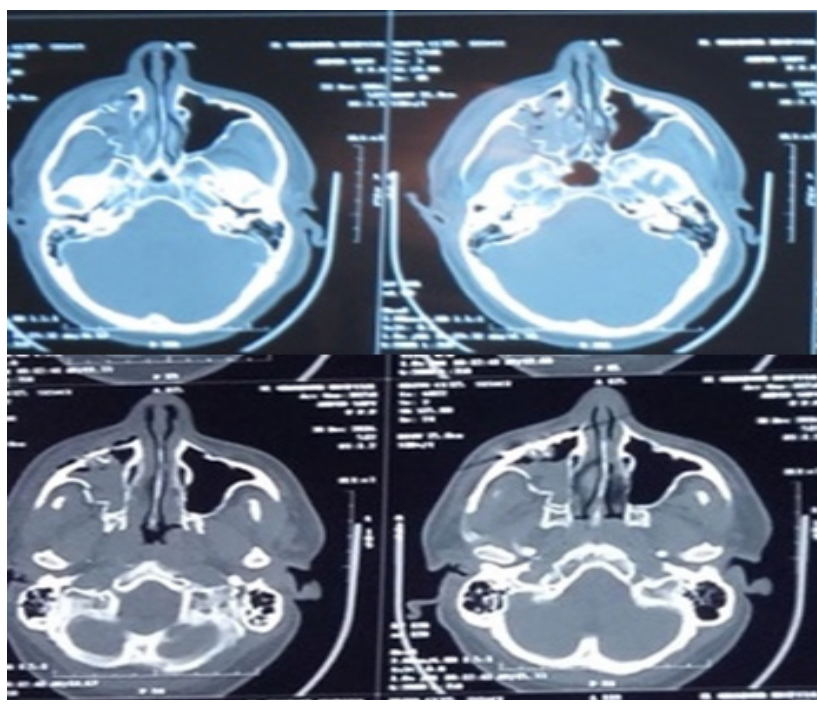

Fig. 2: preoperative and postoperative axial cut C.T.

Time of surgery was recorded in all operation ranged from 12 - 22 minutes average 14.7 minutes \pm 2.83 .

Assessment of nerve function revealed that; postoperative two points discrimination test was negative in all cases (negative 100\%) after 6 months all cases presented with positive results in all cases (positive 100\%) (Table 2)

Table 2: Comparison between periods according to two points discrimination test.

\begin{tabular}{|c|c|c|c|c|c|c|c|}
\hline $\begin{array}{c}\text { Two points } \\
\text { discrimination test }\end{array}$ & Pre-operative & $\begin{array}{c}1 \text { week } \\
\text { post-operative }\end{array}$ & $\begin{array}{c}1 \text { month } \\
\text { post-operative }\end{array}$ & $\begin{array}{c}3 \text { months } \\
\text { post-operative }\end{array}$ & $\begin{array}{c}6 \text { month } \\
\text { post-operative }\end{array}$ & Chi-square test & p-value \\
\hline Yes & $3(27.2 \%)$ & $0(0 \%)$ & $1(9.1 \%)$ & $5(45.5 \%)$ & $11(100 \%)$ & \multirow{3}{*}{28.662} & \multirow{3}{*}{$<0.001 * *$} \\
\hline Nil & $9(72.7 \%)$ & $11(100 \%)$ & $10(90.9 \%)$ & $6(54.5 \%)$ & $0(0 \%)$ & & \\
\hline Total & $11(100 \%)$ & $11(100 \%)$ & $11(100 \%)$ & $11(100 \%)$ & $11(100 \%)$ & & \\
\hline
\end{tabular}

p-value $<0.001 * * \mathrm{HS}$

Assessment of numbness revealed that, all cases where reported with numbness postoperatively,

Table 3: Comparison between periods according to assessment of numbness sensation.

\begin{tabular}{|c|c|c|c|c|c|c|c|}
\hline $\begin{array}{c}\text { Assessment of } \\
\text { numbness sensation }\end{array}$ & Pre-operative & $\begin{array}{c}1 \text { week } \\
\text { post-operative }\end{array}$ & $\begin{array}{c}1 \text { month } \\
\text { post-operative }\end{array}$ & $\begin{array}{c}3 \text { months } \\
\text { post-operative }\end{array}$ & $\begin{array}{c}6 \text { month } \\
\text { post-operative }\end{array}$ & Chi-square test & p-value \\
\hline Yes & $3(27.2 \%)$ & $11(100 \%)$ & $9(81.8 \%)$ & $3(27.3 \%)$ & $0(0 \%)$ & \multirow{3}{*}{27.653} & \multirow{3}{*}{$<0.001 * *$} \\
\hline Nil & $9(72.7 \%)$ & $0(0 \%)$ & $2(18.2 \%)$ & $8(72.7 \%)$ & $11(100 \%)$ & & \\
\hline Total & $11(100 \%)$ & $11(100 \%)$ & $11(100 \%)$ & $11(100 \%)$ & $11(100 \%)$ & & \\
\hline
\end{tabular}

p-value $<0.001 * * \mathrm{HS}$ 
Facial edema was significantly increased in 1 day and 1 week postoperative, after 1 month, it decreased with no significant changes in comparison to postoperative till 6th month. (Figure 3)

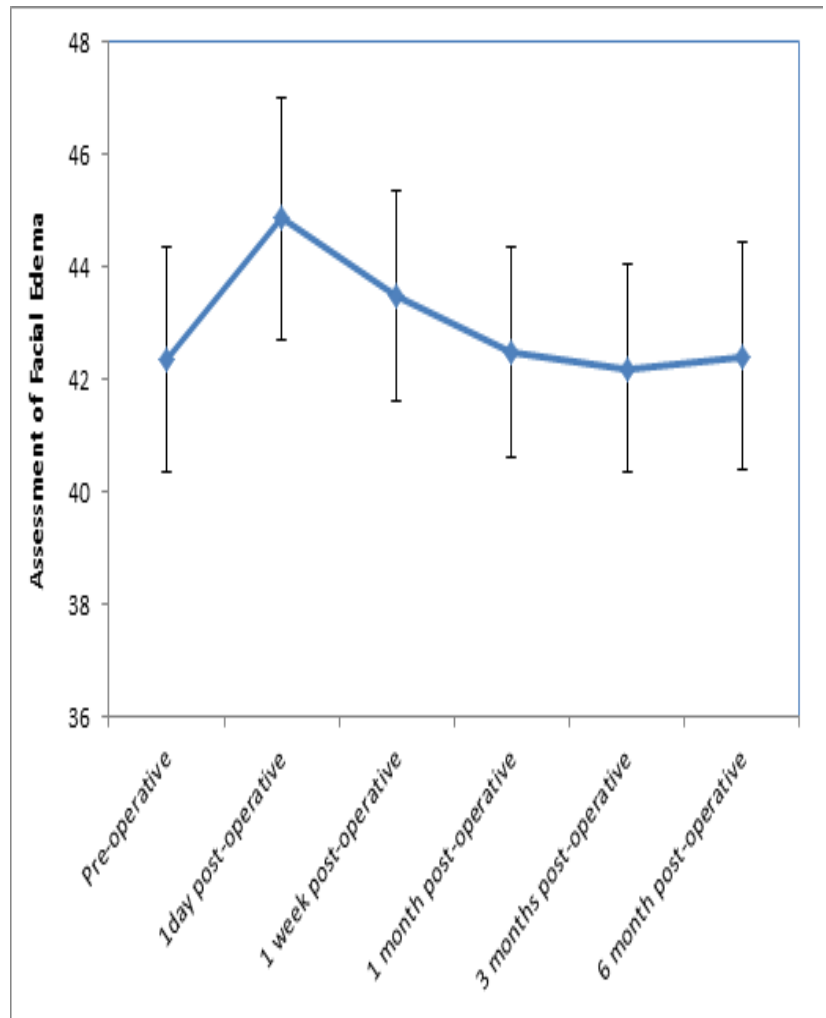

Fig. 3: Comparison between periods according to assessment of facial edema.

\section{DISCUSSION:}

Various approaches have been used and described in literatures to visualize and access the infraorbital rim and the orbital floor over the years. Transconjunctival and transcutaneous like subciliary, subtarsal, and infraorbital approaches are excellent alternatives for surgical treatment of orbital floor and infraorbital rim fractures. ${ }^{[1]}$

However, these techniques involve several complications such as hypertrophic scar formation, scleral show, mild lid edema, keratoconjunctivitis, epiphora, ectropion, lagophthalmos, and nasolacrimal injury. ${ }^{[12]}$

Meta-Analysis published 2009 comparing subtarsal, subciliary, and transconjunctival incisions included seventeen studies representing 2086 patients reported the highest risk of ectropion was in subciliary incisions $(14 \%)$, the risk of entropion was highest transconjunctival incisions (1.5\%), and the risk of hypertrophic scarring was highest in subtarsal incisions $(3.4 \%) .{ }^{[13]}$

Also, it was reported in a recent systematic review and meta-analysis study published in 2017 assessed the incidence of ectropion, entropion, and scleral show that might occur with subciliary and transconjunctival approaches, it becomes clear that the transconjunctival approach has a slight advantage over the subciliary approach. For instance, $14.5 \%$ of patients who underwent subciliary approaches experienced ectropion and/ or scleral show, whereas $8.8 \%$ of patients undergoing a transconjunctival approach experienced ectropion, entropion, or scleral show. ${ }^{[14]}$

In the present study intraoral unilateral vestibular approach were performed with no lower eyelid complications reported in all cases. as there is no manipulation or dissection of lower eyelid in this approach there is no complications.

In a study compared four incisions subciliary, subtarsal, infraorbital and transconjunctival with lateral canthotomy for treatment of orbital rim or floor fractures, it was reported infraorbital, subtarsal, single eye lid incision, provided more rapid access to infraorbital $\operatorname{rim}(8,10,14$ minutes $)$ than did trans-conjunctival incision (22 minutes). ${ }^{[15]}$ In comparison with the current study, the time of surgery was ( average14.7 minutes \pm 2.83 ) indicating that the exposure time of intraoral vestibular approach is comparable with transcutaneous or transconjunctival approaches.

However temporary paresthesia of the infraorbital nerve due to its dissection and elevation is the complication associated with the intraoral approach as it was revealed in results of infraorbital nerve assessment by two points discrimination test and numbness test.

In accordance; it was recommended in a systematic review published in 2018, a variety of clinical neurosensory tests were used in 25 of 28 studies. These included mechanoreceptive tests; light touch sensation, 2-point discrimination, brush directional stroke, and nociceptive tests; sharp-blunt discrimination; and pinprick and thermal testing. ${ }^{[16]}$

The incidence of sensory disturbances in orbitozygomatic complex fractures in the immediate post-trauma period varies from $24 \%$ to $94 \%$ which is in agreement with the present study results $27.2 \%$ however, all cases presented complete resolution of neurosensory function which indicate the advantage for the present technique in decompression around the infraorbital nerve in improving neurosensory functions as the most of patients with orbitozygomatic complex fractures, the fracture line involves the infraorbital foramen. ${ }^{[17]}$

The information revealed by numbness testing was in agreement with two points discrimination test. Both tests were performed in the current study to evaluate infraorbital nerve affection by trauma itself as clarified in the preoperative records or that occurred due to manipulation of the nerve during surgery and although all cases suffered from postoperative negative results, they improved gradually and normal sensation was recovered.

Upon our knowledge There are no available Articles available in literature evaluating the infraorbital nerve affection in intraoral approach for management of infraorbital rim however regard to other approaches in management of zygomaticomaxillary complex fracture 
several articles reported infraorbital nerve affection. Taicher et al compare the incidence of persistent sensory disturbance after recovery from with four treatment methods; closed reduction without fixation, open reduction without fixation, open reduction with support by an intra antral Foley catheter and open with fixation of the zygomaticofrontal process; they reported $70 \%$ patients were free of paresthesia after open reduction and internal fixation. ${ }^{[18]}$ Sakavicius et al reported improvement in $77.3 \%$ of patients after open reduction and internal fixation these findings are in agreement with the results of the current study. ${ }^{[19]}$ A recent study compared the recovery of infraorbital nerve paresthesia following open reduction and internal fixation verses closed reduction in the management of ZMC fractures, it was reported infraorbital nerve recovery in patients treated with open reduction and internal fixation was found $74 \% .{ }^{[20]}$ In comparison with the current study this will be in favour for the intraoral approach for infraorbital rim reduction and fixation as dissection, tunnelling, direct reduction and fixation around the infraorbital nerve were beneficial in decompression and repair to the nerve.

Clinical evaluation of facial edema revealed that there were no significant facial asymmetry or disfigurement resulted after 1 month while it was highly obvious in the first week. Other studies presented moderate surgical edema postoperatively and disappeared by the third to fifth postoperative day. With eyelid approaches patients in both the groups presented with subconjunctival and circumorbital edema. ${ }^{[21,22]}$ presented facial edema in the current approach is referred to the tissue manipulation and dissection to reach the infraorbital rim however, the postoperative edema may be accepted to the patients rather than scaring or multiple incisions as well as intraoral incision is performed for reduction and fixation of zygomaticomaxillary buttress reduction and fixation.

This technique presents some advantages such as less morbidity, fewer complications, and simplicity. On the other hand, its limited to access to the infraorbital floor. After correct reduction of the zygomatic complex through intraoral approach and fixation of zygomaticomaxillary buttress and infraorbital rim represent a rapid and conservative with decreased complication rate and periorbital scarring.

\section{CONCLUSION:}

Intraoral unilateral vestibular approach allows access to the zygomatico-maxillary buttress and infraorbital rim with only 1 incision, optimizes surgical time, decreases complication rates, and avoids periorbital scarring in the patient.

\section{REFERENCES:}

1. Trivellato PF, Arnez MF, Sverzut CE, et al. A retrospective study of zygomatico-orbital complex and/or zygomatic arch fractures over a 71-month period. Dent Traumatol 2011;27:135Y142.
2. Bouguila J, Zairi I, Khonsari RH, et al. Fractured zygoma: a review of 356 cases. Ann Chir Plast Esthet 2008;53:495

3. Kushner GM. Surgical approaches to the infraorbital rim and orbital floor: the case for transconjunctival approach. J Oral Maxillofac Surg 2006;64:108

4. Subramanian, B., Krishnamurthy, S., Suresh Kumar, P. et al. J. Maxillofac. Oral Surg. (2009) 8: 99.

5. Yoon, S. H., \& Lee, J. H. The Reliability of the Transconjunctival Approach for Orbital Exposure: Measurement of Positional Changes in the Lower Eyelid. Archives of Craniofacial Surgery, (2017). 18(4), 249-254.

6. Neovius E, Clarliden S, Farnebo F, Lundgren TK Lower Eyelid Complications in Facial Fracture Surgery. J Craniofac Surg. 2017 ;28(2):391 - 393.

7. Olate S, Lima SM Jr, Sawazaki R, Moreira RW, de Moraes M. Surgical approaches and fixation patterns in zygomatic complexfractures.J Craniofac Surg. 2010 ; 21(4):1213 - 7.

8. Ma YQ1, Zhu SS, Li JH, Luo E, Feng G, Liu Y, $\mathrm{Hu}$ J. Reduction malarplasty using an L-shaped osteotomy through intraoral and sideburns incisions. Aesthetic Plast Surg. 2011 ;35(2): $237-41$.

9. Kumar A, Kale TP. A Comparative Study between the Effect of Combined Local Anesthetic and Low-dose Ketamine with Local Anesthetic on Postoperative Complications after Impacted Third Molar Surgery. The Journal of Contemporary Dental Practice, December 2015;16(12):957 - 96

10. Eliav, E., S. Teich, D. Nitzan, D. Raziq and M. Tal,. Facial arthralgia and myalgia: can they be differentiated by trigeminal sensory assessment? Pain, 2003;104: 481 - 90.

11. Yamashita $M$, Kishibe $M$, Shimada K. Incidence of lower eyelid complications after a transconjunctival approach: influence of repeated incisions. J Craniofac Surg 2014; 25:1183-1186.

12. 12. Salgarelli AC, Bellini P, Landini B, et al. A comparative study of different approaches in the treatment of orbital trauma: an experience based on 274 cases. Oral Maxillofac Surg 2010;14:23-27

13. Ridgway EB, Chen C, Colakoglu S, Gautam S, Lee BT. The incidence of lower eyelid malposition after facial fracture repair: a retrospective study and meta-analysis comparing subtarsal, subciliary, and transconjunctival incisions. Plast Reconstr Surg 2009;124:1578-1586.

14. Al-Moraissi EA, et al., Subciliary vs. 
transconjunctival approach for the management of orbital floor and periorbital fractures: A systematic review and meta-analysis, Journal of CranioMaxillo-Facial Surgery 2017;45(10):1647 - 154.

15. Subramanian B, Krishnamurthy S, Suresh Kumar P, Saravanan B, Padhmanabhan M. Comparison of various approaches for exposure of infraorbital rim fractures of zygoma. J Maxillofac Oral Surg. 2009 Jun;8(2):99 - 102.

16. Devine, Maria et al. Identifying criteria for diagnosis of post-traumatic pain and altered sensation of the maxillary and mandibular branches of the trigeminal nerve: a systematic review Oral Surgery, Oral Medicine, Oral Pathology and Oral Radiology, Volume 125, Issue 6, 526 - 540

17. Taicher, S., N. Ardekian, Y. Samet, I. Shoshani and I. Kaffe, 1993. Recovery of the infraorbital nerve after zygomatic complex fractures: a preliminary study of different treatment methods. Int. J. Oral Maxillofac. Surg., 22: 339 - 341

18. Taicher S, Ardekian L, Samet N. Recovery of the infraorbital nerve after zygomatic complex fractures: a preliminary study of different treatment methods. Int J Oral Maxillofac Surg. 1993;22(6):339 - 41.

19. Sakavicius D, Juodzbalys G, Kubilius R, Sabalys P. Investigation of infraorbital nerve injury following zygomaticomaxillary complex fractures. J oral Rehab. 2008;35: 903 - 16.

20. Ishaq Y, Noor M, Anwar MA. Comparison of infraorbital nerve recovery after open and closed reduction of zygomaticomaxillary complex fractures. Int J Otorhinolaryngol Head Neck Surg $2018 ; 4: 613-7$.

21. Prathibha Sridhar, Shubha Sandeep, Kavitha Prasad, Lalitha R. M. , Ranganath K and Sejal Munoyath K. Comparative Evaluation of Single Point Fixation at Zygomatic Buttress and Fronto Zygomatic Rim in Zygomatic Complex Fractures -A Prospective Study. Journal of Dental \& Orofacial Research 2017:13 (02) :27 - 39.

22. Aleem MA, Nasyam FA, Parameshwar Reddy KR, Karpe T, Singh T, Shailaja AB. Management of infraorbital rim and orbital floor fractures: A comparison of subciliary and infraorbital approaches. J Int Oral Health 2017;9:65 - 70. 